\title{
EchoGéo
}

\section{Révolution et recherche : développement durable et transformations sociales à Cuba}

\section{Sabrina Doyon}

\section{(2) OpenEdition}

1 Journals

Édition électronique

URL : https://journals.openedition.org/echogeo/11731

DOI : 10.4000/echogeo.11731

ISSN : 1963-1197

\section{Éditeur}

Pôle de recherche pour l'organisation et la diffusion de l'information géographique (CNRS UMR 8586)

\section{Référence électronique}

Sabrina Doyon, « Révolution et recherche : développement durable et transformations sociales à Cuba », EchoGéo [En ligne], 12 | 2010, mis en ligne le 31 mai 2010, consulté le 01 août 2021. URL: http://journals.openedition.org/echogeo/11731; DOI : https://doi.org/10.4000/echogeo.11731

Ce document a été généré automatiquement le 1 août 2021.

EchoGéo est mis à disposition selon les termes de la licence Creative Commons Attribution - Pas d'Utilisation Commerciale - Pas de Modification 4.0 International (CC BY-NC-ND) 


\title{
Révolution et recherche : développement durable et transformations sociales à Cuba
}

\author{
Sabrina Doyon
}

\section{Introduction}

1 L'appel, en mai 2009, des médias officiels cubains à la population fut sans équivoque : "Ahorro o muerte ». L'île est gravement affectée par la crise économique mondiale, obligeant les divers secteurs de production ainsi que les habitants à s'adapter à d'importantes nouvelles restrictions, dont la diminution de l'offre de nourriture, les coupures d'électricité - les « apagones » - et les fermetures temporaires d'usines. Dans ce contexte, le financement par l'État du secteur de l'éducation et de la recherche est compromis, une situation exacerbée dans la tourmente actuelle, mais qui existe cependant depuis la mise en place de la " période spéciale en temps de paix ».

2 Cette situation pourrait être critique pour l'État socialiste cubain, car, comme le notent entre autres Blumm (2008) et Ninetto (2005), l'éducation et la recherche scientifique sont des lieux privilégiés pour la construction de l'identité et de la conscience révolutionnaire dans les Ėtats socialistes et postsocialistes ; l'État a su y instiller ses valeurs sociales, économiques et politiques. On constate que, malgré les difficultés économiques des dernières décennies, l'État cubain a su préserver ce secteur grâce, entre autres, aux transferts d'argent provenant de l'aide internationale. Cet article analyse, par un exemple ethnographique, la manière dont l'éducation et la recherche scientifique en environnement furent maintenues par la mise en place d'un projet de développement durable financé par une organisation d'aide internationale au développement - le Centre de recherche pour le développement international du Canada.

3 L'examen de cette situation nous amène à questionner les mécanismes de la construction de la révolution dans cette ère "post-Castro». Comment se vit la 
révolution dans le contexte des transformations apportées au système, notamment à travers l'insertion de capital étranger que l'on observe entre autres dans la recherche scientifique? Est-ce que ces changements érodent la révolution et la conscience du revolucionario? Sont-ils des signes avant-coureurs d'une mort annoncée par plusieurs ou des stratégies de « survie révolutionnaire »?

\section{Révolution et transformations socioéconomiques}

4 Cette étude s'inscrit dans la foulée d'un nombre croissant de recherches qui analysent les interprétations et les représentations de la révolution et de l'identité révolutionnaire, tant du point de vue des individus que des institutions (se référer entre autres à Brotherton, 2003, 2005 ; Carter, 2000 ; Daniel, 1995 ; Doyon, 2003, 2005 ; Fernández, 1996 ; Forrest, 1999 ; Fosado, 2004 ; Gropas, 2007 ; Hernandez-Reguant, 2002 ; Holbraad, 2002 ; Palmié, 2004 ; Perry, 2004 ; Powell, 2008 ; Premat, 2004, 2009 ; Roland, 2006 ; Rosendhal, 1997). Les prémisses de la recherche s'ancrent dans une vision plurielle et rhizomique de la révolution où l'État n'est pas considéré comme un acteur monolithique et univoque et où les habitants ne sont pas que des sujets passifs des politiques et des réformes mises en place (Doyon et Brotherton, 2008). Comme le révèle l'approche ethnographique, la révolution est constituée d'une multitude d'interprétations, tant individuelles qu'institutionnelles, qui la transforment dans le cadre d'un monde globalisé. Elle est le lieu de négociations subtiles, de paradoxes et d'ambivalences qui permettent à ses acteurs d'y créer du sens.

5 Les transferts d'argent dans le secteur de l'éducation et de la recherche - et l'implantation de nouveaux concepts qui les accompagnent, tel le développement durable - constituent un de ces mécanismes de transformation et d'ajustement de la révolution. Ces changements ont parfois été en contradiction avec les principes révolutionnaires. C'est le cas avec certaines des réformes mises en place depuis 1990 : restauration de la capacité d'importation et stimulation de l'offre domestique, augmentation dans la capacité de réponse de l'économie au marché mondial, recherche de capital étranger et de technologies, permission d'effectuer des ventes libres sur le marché de certains surplus de production, d'artisanat et de certains biens manufacturés, création de catégories « d'emploi autonome " permis par l'État couvrant plus d'une centaine d'occupations, enregistrement et taxation d'activités de location privées (comme des chambres chez l'habitant pour touristes) (E.I.U. 1997). Il va sans dire que ces changements ont bénéficié aux habitants ayant accès aux devises fortes, soit grâce à des transferts d'argent ou des activités touristiques, créant de nouvelles et importantes inégalités au sein de la population. Cette dynamique crée un sentiment d'injustice au sein de la société qui peut potentiellement affecter la conscience révolutionnaire des habitants.

Plusieurs de ces activités, bien que faisant partie de ce qu'Eckstein appelle la «culture de l'illégalité » (1997: 148), furent incorporées dans le programme des réformes économiques promues par l'État. Castro soutient que ces stratégies économiques furent planifiées dans le but de sauver la révolution, arguant que la marchandisation croissante et la privatisation "ne violent aucun principe du socialisme" (Granma Weekley Review, 1991 : 15). Le gouvernement adapte ainsi sa façon d'exercer le pouvoir. Il cherche à repenser la manière de préserver les principes de souveraineté et d'égalité $\mathrm{du}$ socialisme dans un contexte de transformations socioéconomiques globales 
marquant la vie quotidienne des habitants. Certaines analyses avancent que ces transformations, plus particulièrement les changements des politiques économiques, seraient le talon d'Achille du socialisme cubain. Cette perspective sous-entend l'idée que le système ait une finalité, qui serait connue et déterminée d'avance, reposant inévitablement dans l'accession au néolibéralisme, entendu comme un principe de «bonne gouvernance» et de libre marché. Comme le développent certains auteurs dans leur analyse de la transition et des sociétés "post-post-transition" (Buyandelgeriyn, 2008), le cas de Cuba témoigne plutôt de la complexité de cette situation, car les pratiques informelles, liées entre autres à l'insertion de capitaux étrangers ainsi qu'aux interprétations de nouveaux concepts tels le développement durable, contribuent à reconfigurer l'État socialiste et à recréer la révolution dans les pratiques quotidiennes de ses sujets. Ce processus de construction peut-il menacer la révolution? L'insertion de concepts tels que le développement durable, proposant dans leur formulation initiale des valeurs d'égalité ainsi que de justice sociale et générationnelle, proches de celles du socialisme, tout en étant solidement ancrées dans les logiques néolibérales contemporaines, favorise-t-elle ou mine-t-elle la révolution?

7 Les transferts d'argent par la filière de l'aide au développement représentent-ils dans le secteur scientifique un moyen de préserver la révolution et ses acquis par le maintien de ses programmes de recherche? Comment cette stratégie s'appuie-t-elle sur l'introduction et la manipulation d'un concept particulier, le développement durable, agissant comme un outil, utilisé tant par l'État que la société civile, pour défendre, justifier, interpréter, et négocier la révolution et ses transformations?

8 Afin d'aborder ces questions, les liens unissant l'éducation, la recherche scientifique et l'aide au développement dans le cas d'un projet de développement durable mené dans la province de Pinar del Río seront présentés. La mise en place de cette initiative et ses implications aux niveaux institutionnel et individuel seront examinées. L'analyse se fonde sur des recherches ethnographiques effectuées de juin 2001 à septembre 2002, et sur des séjours annuels pendant lesquels des entrevues furent effectuées avec des habitants de communautés côtières, des chercheurs et des représentants du gouvernement, plus particulièrement du Ministère des sciences, des technologies et de l'environnement (CITMA), dans la province de Pinar del Río.

\section{Révolution, science et aide au développement}

9 L'éducation est un des fleurons de la révolution cubaine. Le pays peut se targuer d'avoir un taux d'alphabétisation enviable de $96 \%$. Si l'éducation primaire et secondaire est au cœur des fondements de la révolution, l'éducation et la recherche post-secondaire n'en constituent pas moins un de ses éléments centraux. Cuba comptait en 2006 près de 300000 inscrits aux cycles supérieurs, principalement en sciences humaines et en médecine, avec plus de 8000 graduations cette même année. La recherche scientifique est valorisée, le pays se démarquant entre autres par sa production scientifique dans le domaine des biotechnologies. Pour l'État, cette situation témoigne clairement du succès de la révolution. Malgré cela, la pérennité de l'éducation supérieure et de la recherche scientifique est compromise depuis la " période spéciale ${ }^{1}$ dans tous les secteurs qui ne sont pas relatifs à la santé, et plus particulièrement dans les secteurs des sciences humaines et sociales. De nombreux domaines sont sous-financés, plusieurs chercheurs doivent exercer en même temps d'autres emplois sans lien avec le milieu académique 
pour subvenir à leurs besoins, et de plus en plus de jeunes choisissent de ne plus fréquenter les universités car ils disent qu'elles «leur ferment des portes $»^{2}$. Dans ce contexte, le gouvernement, qui n'a désormais plus les moyens de financer ce secteur comme auparavant, pousse les chercheurs et les institutions universitaires à trouver des fonds dans divers projets de développement internationaux, à devenir des "entrepreneurs socialistes " ${ }^{3}$, enfreignant des principes clés de la révolution et menaçant son intégrité. Comment la révolution peut-elle perdurer dans le cadre de ces pratiques capitalistes?

Dans le secteur environnemental, l'État a pallié ces difficultés en développant des initiatives conjointes avec des programmes d'aide au développement, promouvant entre autres le concept de développement durable. ${ }^{4}$ C'est cette stratégie, combinant l'entrepreunariat individuel et l'accès à des fonds étrangers, qu'adoptèrent les cinq chercheurs principaux du projet de Développement Durable des Ressources Côtières (MASOREC Manejo sostenible de los recursos costeros) financé par le Centre de développement international du Canada (CRDI). Les chercheurs, accompagnés d'une dizaine de collaborateurs et d'autant d'étudiants, ont mené de 1999 à 2002 un projet de développement durable sur la côte sud de la province de Pinar del Río. Ce projet visait la préservation et le reboisement de la forêt de mangroves avoisinant les villages de cette région. Celle-ci avait subi une importante dégradation, conséquence de la " période spéciale » durant laquelle la population avait été poussée à décimer les forêts avoisinantes pour produire du charbon en l'absence d'autres combustibles autrefois fournis par l'État (Doyon, 2005a). Ce projet, d'une valeur de $20000 \$$ répartis sur trois ans, s'ancrait directement dans les préoccupations scientifiques des chercheurs locaux et fut entièrement mené par ces derniers ; en contrepartie, ils devaient aussi promouvoir l'objectif principal du CRDI, soit le développement durable des ressources par la recherche interdisciplinaire et la participation locale des communautés touchées par la dégradation environnementale.

11 Le groupe MASOREC a ainsi tenté de répondre aux objectifs scientifiques de l'organisme subventionnaire, mais la subvention a surtout permis de financer la recherche universitaire et, d'une certaine manière l'université, désargentées. L'argent, géré par le service des finances de l'université, a été dépensé en partie pour l'achat d'équipement de recherche, de fourniture de bureau de base, de branchement à Internet, mais aussi en déplacements et per diem ${ }^{5}$. Il a rendu possible pour les cinq chercheurs principaux la collecte des informations qui leur ont chacun permis de terminer leurs thèses de doctorat. Les données ont permis aux collaborateurs de poursuivre leurs recherches et aux étudiants de maîtrise et de baccalauréat impliqués de terminer leurs programmes d'étude. Ainsi, l'intérêt de chacun des chercheurs était davantage orienté vers leurs recherches individuelles que sur le projet en tant que tel, et son objectif d'interdisciplinarité fut difficile à réaliser, comme nous l'a avoué un des chercheurs : «l'interdisciplinarité est difficile à atteindre et nous ne l'avons pas atteint à la perfection, il n'y eut pas une grande intégration, mais nous avons travaillé pour l'atteindre».

12 Le volet communautaire de la recherche n'a pas été non plus exploité de manière optimale. Les chercheurs en sciences naturelles n'étaient d'abord pas formés à la recherche participative, et l'objectif de leurs séjours sur le terrain était en tout premier lieu de collecter des données biophysiques sur la forêt et non pas d'élaborer un projet communautaire. De même, les ateliers participatifs et les activités de nettoyage des 
mangroves ne furent pas effectués dans l'esprit de la recherche participative, qui suppose l'intégration des populations dans la définition et la résolution de problèmes de développement durable. Comme le mentionnait un chercheur à propos des habitants du village où avait lieu le projet : «ils ont une méconnaissance totale de l'écosystème de mangroves "; un autre est plus cinglant : « ils ne se sont pas assis pour analyser, ils ne savent pas comment utiliser les ressources, ils n'ont pas conscience jusqu'à quel point ils peuvent le faire, ils vivent dans un lieu entouré de mangroves et en plus ils vivent de ces ressources, je crois qu'ils ont fait un usage irrationnel et ils ne prennent pas en compte les conséquences de la surexploitation » (voir Doyon 2005b pour une discussion sur le savoir local).

13 Les chercheurs ont toutefois réalisé ces activités en toute bonne foi et dans la mesure de leurs capacités. Alors que le projet devait promouvoir en premier lieu la gestion communautaire des ressources côtières, il est possible d'avancer que c'est le développement institutionnel qui a plutôt pris le dessus. Ce genre de situation n'est pas exclusif à Cuba, mais dans ce cas précis, il contribue directement au maintien de la révolution et dans une certaine mesure de ses idéologies. En effet, les fonds étrangers ont permis de subvenir et de maintenir la recherche universitaire en environnement (un domaine important puisque les inscriptions en sciences naturelles sont à la baisse selon le Ministère de l'éducation), de produire des diplômés, de regarnir les laboratoires, tout en investissant dans un domaine d'intérêt économique pour l'État. Ces recherches permettent de protéger les mangroves et les langoustes offrant pour cette seule région des profits de 15 millions de dollars US par an (Rodriguez, 2003). Elles sont donc en phase avec les priorités nationales de développement. Ce secteur est de cette façon subventionné par des devises étrangères, provenant du système capitaliste international, qui lui permettent de se reproduire. Voilà une approche en apparence contradictoire avec les principes de la révolution, mais qui permet à cette dernière de se maintenir de façon pragmatique. L'approche ethnographique permet d'examiner comment ces initiatives sont reprises et utilisées par les acteurs locaux et dans quelle mesure elles permettent d'interpréter et de vivre les idéologies révolutionnaires.

\section{Développement durable : interprétations d'un nouveau discours révolutionnaire}

Les initiatives d'aide internationale permettent, par leur subvention de recherche en devises fortes, l'insertion d'une nouvelle forme de capital sur l'île, prenant place dans l'espace laissé béant par la crise économique de la "période spéciale ». Fondées sur le concept de développement durable, ces initiatives ne sont toutefois pas purement philanthropiques et neutres. Comme le discutent notamment Escobar (1995) et Sachs (1999), le développement durable a différentes visées qui s'ancrent généralement dans des intérêts économiques très concrets instrumentalisant les enjeux de justice sociale et de protection environnementale qu'il promeut. Des flous sémantiques sont entretenus dans l'évocation et la mise en place de ses principes permettant à ses promoteurs de réaliser leurs intérêts. Les initiatives d'aide internationale au développement environnemental ont pour objectif d'améliorer la situation environnementale, certes, mais aussi d'implanter des valeurs de «bonne gouvernance ", de démocratie et de néo-libéralisme où elles sont mises en place, facilitant par la suite la réalisation de leurs objectifs économiques. Usant de la 
flexibilité des significations accordées au concept de développement durable, les politiques et les idéologies de l'État cubain se faufilent dans les dédales de cette logique développementaliste et instrumentalisent ces initiatives à leur tour. L'approche ethnographique permet d'examiner dans quelle mesure ces appropriations permettent de maintenir ou de renverser les valeurs révolutionnaires.

15 C'est dans la foulée du sommet de Rio et de la période spéciale que l'État cubain a repris à son compte le concept de développement durable. Terme fourre-tout, le développement durable est utilisé dans le contexte cubain pour décrire une multitude de choses: des initiatives locales de protection d'espèces endémiques emblématiques par des enfants d'une école primaire diffusées au téléjournal du soir aussi bien que le développement d'infrastructures touristiques. Les discours étatiques autour du développement durable s'inscrivent généralement dans la perspective du développement économique, et permettent de justifier dans ce concept « rassembleur » et globalisant différentes initiatives nationales, parfois contradictoires et pas toujours pérennes. Le discours autour du développement durable tel que déployé par l'État cubain encapsule aussi les valeurs sociales de la révolution - justice, équité pour les générations futures, progrès- et en ce sens il devient un autre emblème des valeurs cubaines, représentant un nouveau projet de la société révolutionnaire du $21^{\mathrm{ème}}$ siècle où chacun est amené à participer. Le développement durable devient alors justifiable du point de vue de la révolution, il s'institutionnalise.

Reflétant sa nature changeante sur la scène discursive internationale, les significations du concept de développement durable varient entre les organismes de développement, le gouvernement cubain, les chercheurs, et la population visée par ces initiatives. Le développement durable incarne une multiplicité de logiques qui correspondent à autant de perspectives et d'intérêts au sein de la société, et ce tant pour l'État et ses différents ministères et entreprises, que pour la population. Tout comme les fonds distribués par les programmes d'aide au développement, le concept de développement durable est aussi réapproprié par les sujets. Il est utilisé localement par ces derniers comme un nouveau support logique et discursif pour formuler des demandes et des récriminations dans un vocabulaire sanctionné par l'État. Dans le contexte de la " période spéciale », auquel s'ajoute celui de la crise financière actuelle souvent difficile à vivre au quotidien pour la population, le développement durable, proposé à la fois par l'État et l'aide internationale, devient un nouvel outil interprétatif «multifonction » pour négocier la révolution et l'adapter, ouvrant de nouvelles portes à ce que signifie être Cubain. Le développement durable devient pour la population une «banque de données » discursive et pragmatique permettant de justifier différentes actions et positions.

17 Pour les chercheurs du projet MASOREC, le développement durable est un idéal à atteindre à travers l'étincelle de la conscience, la chispa de la conciencia ; comme un des chercheurs principal le souligne : «je crois que la situation environnementale est très favorable pour le futur. Un monde meilleur est possible et on peut atteindre le développement durable, ou tout du moins nous nous dirigeons dans cette direction. Le plus important c'est la conscience des gens, l'étincelle est dans les esprits ». Ces chercheurs butent cependant sur des politiques contradictoires face à une situation environnementale préoccupante. Les ressources naturelles se sont grandement détériorées depuis la révolution (Wotzkow, 1998). L'État, à travers ses différents ministères (sucre, pêches, mines, tourisme, forêts), a toujours favorisé une logique 
d'exploitation et d'extraction des ressources à grande échelle ${ }^{6}$. Il ne s'est pas préoccupé des conséquences destructrices des pratiques d'extraction, agissant sur l'environnement tel un conquérant devant dominer la nature (Díaz-Briquets et Perez Lopez, 2000).) Ce n'est que récemment que les enjeux environnementaux furent reconnus par le gouvernement, virage amorcé lors de la conférence de Rio en 1992. Malgré un discours vantant les mérites du développement durable, les actions entreprises par l'État demeurent encore largement destructrices, entre autres dans les régions côtières avec les développements touristiques de masse. ${ }^{7}$ Bien que ne s'inscrivant volontairement pas à faux avec le discours d'État, les chercheurs ont une réelle préoccupation pour la conservation de l'environnement. Le concept de développement durable leur permet d'avoir un nouvel objectif formulé et entériné par l'État.

Ce concept a permis aux chercheurs d'entamer un dialogue avec des représentants des entreprises étatiques d'exploitation des ressources naturelles (forestière, agraire et halieutique) alors qu'ils réalisaient leurs recherches et collectaient leurs données. Les chercheurs proposent à leurs dirigeants de transformer leurs pratiques d'extraction destructrices afin de préserver l'environnement. L'utilisation du concept de développement durable leur a permis de tenir ce discours "critique » sans qu'ils ne soient accusés d'être contre-révolutionnaires. Un chercheur nous dit à propos de leurs négociations avec les représentants de l'usine de pêche: "ils ne peuvent pas faire comme si ils étaient dans un monde à part, ils doivent se lier avec le reste et comprendre l'importance des mangroves pour leurs activités »; en ce qui concerne l'entreprise sylvicole: «il y a eu une absence totale de gestion des mangroves par l'entreprise forestière, il n'y a de gestion d'aucun type, et c'est maintenant qu'on commence à prendre en compte la loi forestière ». Malgré ces critiques, les chercheurs ne peuvent pas être accusés d'aller à l'encontre des intérêts du pays et de la révolution car ils s'appuient sur le terme du développement durable. Ce concept, institutionnalisé par l'État qui en a fait un objectif politique, permet aux chercheurs de proposer de nouvelles idées dans des secteurs où la production prime généralement sur la conservation.

19 Comme le note Blum $(2008,145)$ les discours produits dans les sociétés dotées d'un État contrôlant doivent être compris dans leur contexte historique et idéologique: "Consequently, any outside observer must learn to interpret everyday talk or discourse as complex negotiation between the state and the ways that individual Cubans interpret and reinterpret reality(ies). Moreover, the dynamic conception of knowledge, as always already partial, situated, and actively produced, accounts for not only "semantic" (literal) meanings for which ideological discourse stands but also for "pragmatic" meanings that emerge in discourse as situated activity.» Le développement durable, concept exploité dans les recherches subventionnées par le développement international, offre aux chercheurs une nouvelle façon d'envisager et de construire la révolution. De même, il est compris et utilisé de diverses manières par les habitants de la communauté de Las Canas, située au sud de la province de Pinar del Río.

20 Las Canas compte 250 habitants s'adonnant principalement à la pêche et à la fabrication de charbon (cf. Doyon, 2005a). Ces derniers ont accueilli favorablement les scientifiques et leur projet de développement durable. En plus du nouveau réseau de socios que sont devenus pour eux les chercheurs du projet, ce qui les a surtout séduits 
dans ce dernier, ce ne sont pas tant les principes de gestion participative ou même de conservation des mangroves, mais bien le concept de développement durable et les possibilités de jeu sémantique qu'il leur offre. Plutôt que de concevoir le développement durable comme un outil de conservation des ressources naturelles, comme le proposent les chercheurs, ils y ont vu un moyen d'aborder le développement local. Depuis la "période spéciale ", plusieurs maisons se sont écroulées, les services touristiques sont fermés et la plage est impraticable en raison de l'érosion et de la contamination par les égouts. Le développement durable représente pour les habitants la possibilité de proposer des projets qui les intéressent, comme l'écotourisme, dans la logique du développement durable qui leur fut enseignée, gérés par la communauté à qui reviendraient les profits, une idée contraire aux principes révolutionnaires. Bien que cette proposition ne soit pas conforme aux principes de la révolution, parce qu'elle sous-entend l'entreprise privée et le profit, il est possible pour les habitants de l'évoquer sans courir de risques puisqu'elle s'inscrit dans le cadre du développement durable, concept accepté par l'État.

Les membres de la communauté voient le développement durable comme une possibilité d'améliorer leurs conditions de vie en combinant les apports du capital international et de l'État. De même, la plate-forme que leur offre le discours hétéroclite du développement durable permet aux habitants de Las Canas de témoigner de leurs conditions de vie difficiles, de leur pauvreté et de leurs luttes quotidiennes dans des termes acceptés par l'État, et même considérés comme révolutionnaires. Ils peuvent s'exprimer et dans une certaine mesure critiquer leur situation sans courir le risque que leurs propos soient mal interprétés et sans remettre le gouvernement en cause. Le développement durable offre une nouvelle passerelle au déploiement de leur capacité d'agir et d'interpréter, et permet aux sujets de négocier la définition de la révolution et du socialisme.

\section{Conclusion}

Si la révolution se vit au quotidien dans un contexte "d'économie ou de mort ", les changements que cette situation a provoqués ouvrent la porte à diverses constructions individuelles et collectives qui ont permis jusqu'à présent aux Cubains de survivre et à la révolution de se maintenir, de se transformer et de se reproduire. Alors que l'État ne peut plus subvenir aux besoins de sa population comme il a cherché à le faire pendant près de 30 ans, il utilise les nouvelles formes d'insertion de devises fortes pour maintenir et consolider des acquis qu'il a défendus, entre autres l'éducation et la recherche. Les entrées de devises sous différentes formes lui permettent de maintenir certaines de ces pierres angulaires de la révolution. À Cuba, la diversification des entrées de capitaux étrangers depuis plus d'une décennie n'a pas contribué à détruire ou miner le système en place, qui se revendique toujours du socialisme malgré ses contradictions et ses failles. Au contraire, elle produit des configurations de l'État dans lesquelles les citoyens développent de nouvelles interprétations et expériences de la révolution.

Les transferts de capitaux ne sont pas les seuls éléments permettant le déploiement de ce processus. De nouvelles idées et concepts peuvent aussi jouer ce rôle. Celui du développement durable est un des outils utilisés dans cette logique. L'ambiguïté propre à ce concept, existant tant au niveau global que dans son interprétation nationale, est 
en phase avec l'ambivalence de l'Hombre novisimo (Frederik, 2005), qui combine les caractéristiques de l'homme nouveau du Ché avec les flous et les paradoxes de système actuel. Cet hombre novisimo peut désormais exprimer davantage son individualité. Le développement durable, concept ancré dans le néolibéralisme et la marchandisation de l'environnement, permet paradoxalement au nouveau révolutionnaire de l'ère «postCastro » de vivre et d'inventer sa révolution.

Ces espaces de contradictions et de paradoxes entre les pratiques et les discours des individus et des institutions peuvent être d'autres clés pour comprendre la portée de la révolution aujourd'hui, et les manières par lesquelles les individus y créent du sens. Dans cette perspective, il serait intéressant dans les études portant sur les transformations contemporaines de la révolution cubaine d'examiner plus en profondeur le processus de « vernacularisation » (Engle Merry, 2006) et les implications de l'insertion de nouveaux concepts provenant de la scène globale, tel le développement durable, qui accompagnent souvent les transferts d'argent dans les négociations de ces espaces de contradictions.

\section{BIBLIOGRAPHIE}

Alarcon de Quesada R., 1995. Cuba: Law number (77) Foreign Investment Act.Official Gazette, Special Issue No. 3.

Blum D., 2008. Socialist Consciousness Raising and Cuba's School to the Countryside Program. Anthropology and Education Quarterly, 39, 2, p. 141-160.

Brotherton P.S., 2003. The Pragmatic State: Socialist Health Policy, State Power, and Individual Bodily Practices in Havana, Cuba. Thèse, Montréal, Université McGill.

Brotherton P.S., 2005. Macroeconomic Change and the Biopolitics of Health in Cuba's Special Period. Journal of Latin American Anthropology, 10, 2, p. 339-369.

Buyandelgeriyn M., 2008. Post-Post-Transition theories: walking on multiple paths. Annual Review of Anthropology, 37, p. 237-350.

Carter T., 2000. Playing Hardball: Constructions of Cuban Identity. Thèse, Albuquerque, Université du Nouveau Mexique.

Daniel Y., 1995. Rumba: Dance and Social Change in Contemporary Cuba. Bloomington, Indiana University Press, 208 p.

Díaz-Briquets S. et J. Perez Lopez, 2000. Conquering Nature: The Environmental Legacy of Socialism in Cuba. Pittsburgh, University of Pittsburgh Press, 344 p.

Doyon S., 2003. L'environnement « révolutionnaire »: Pratiques, discours et dynamique socioenvironnementale de la gestion des ressources côtières à Cuba. Thèse, Montréal, Université McGill.

Doyon S., 2005a. Une révolution à sa mesure : Diversité des pratiques environnementales communautaires à Cuba. Anthropologie et sociétés, 29, 1, p. 121-144. 
Doyon S.,2005b. Savoir environnemental local et scientifique : Entre la révolution, la gouvernance nationale et le développement durable à Cuba. Vertigo, 6, 2, p. 4-11.

Doyon S., 2007. Fishing for the Revolution. Transformations and Adaptations in Cuban Fisheries. Mast, 6, 1, p. 83-108.

Doyon S. et P.S. Brotherton, 2008. Les redéfinitions d'une révolution. Pratiques et politiques dans les secteurs de la santé et de l'environnement à Cuba. Anthropologie et sociétés, 32, 1-2, p. 193-216.

Doyon S. et P.S. Brotherton, 1997. The Limits of Socialism in a Capitalist World Economy: Cuba Since the Collapse of the Soviet Bloc, inCenteno M.A. et M. Font (dir.), Toward a New Cuba? Legacies of a Revolution. London, Boulder, p. 135-150.

Economist Intelligence Unit (E.I.U.), 1997, Cuba: A Country Profile.

Engle Merry S., 2006. Transnational Human Rights and Local Activism: Mapping the Middle. American Anthropologist, 108, 1, p. 38-51.

Escobar, A., 1995. Encountering Developement. The Making and Unmaking of the Third World. Princeton, Princeton University Press, 320 p.

Fernández N.T., 1996. The Color of Love: Young Inter-racial Couples in Cuba. Latin American Perspectives, 23, 1, p. 99-117.

Forrest D.P., 1999. Bichos, Maricones and Pingueros: an Ethnographic Study of Maleness and Scarcity in Contemporary Socialist Cuba, Thèse, Londres, Université de Londre.

Fosado G.C., 2004. The Exchange of Sex for Money in Contemporary Cuba: Masculinity, Ambiguity, and Love. Thèse, Détroit, Université du Michigan.

Frederik L.A., 2005. Cuba's National Characters: Setting the Stage for the Hombre Novisimo. Journal of Latin American Anthropology, 10, 2, p. 401-436.

Gropas M., 2007. The Repatriotization of Revolutionary Ideology and Mnemonic Landscape in Present-Day Havana. Current Anthropology, 48, 4, p. 531-549.

Hernandez-Reguant A., 2002. Radio Taino and the Globalization of the Cuban Culture Industries. Thèse, Chicago, Université de Chicago.

Holbraad M., 2002. Belief in necessity: Ifá Divination and Money in Contemporary Havana. Thèse, Cambridge, Université de Cambridge.

Morris E., 2008. Cuba's New Relationship with Foreign Capital: Economic Policy-Making Since 1990. Journal of Latin American Studies, 40, p. 769-792.

Ninetto A., 2005. "An Island of Socialism in a Capitalist Country": Postswocialist Russian Science and the Culture of the State. Ethnos, 70, 4, p. 443-464.

Palmié S., 2004. Fascinans or Tremendum? Permutations of the State, the Body, and the Divine in Late-Twentieth Century Havana. New West Indian Guide, 78, 3-4, p. 229-268.

Perry M.D., 2004. Los raperos: Rap, race, and Social Transformation in Contemporary Cuba. Thèse, Austin, Université du Texas.

Powell K., 2008. Neoliberalism, the Special Period and Solidarity in Cuba. Critique of Anthropology, 28, 2, p. 177-197.

Premat A., 2009. State Power, Private Plots and the Greening of Havana's Urban Agriculture Movement. City and Society, 21, 1, p. 28-57. 
Premat A., 2004. Cuban Counterpoint of the Public and the Private: Reflections on the Making of Urban Agriculture Sites in Havana, Cuba. Thèse, Toronto, Université de York.

Rodrígez G., 2003. Base para el Manejo Sostenible de un Bosque de Manglar en Estado de Deterioro.

Thèse, Pinar del Rio, Université de Pinar del Rio, San Vicente del Raspeig Université Alicante.

Roland L.K., 2006. Tourism and the Negrification of Cuban Identity. Transforming Anthropology, 14, p. 151-62.

Rosendhal M., 1997. Inside the Revolution: Everyday Life in Socialist Cuba. Ithaca and London, Cornell University Press, 208 p.

Sachs W. 1999. Planet Dialectics. Exploration in Environment and Development. London, Zed Books, $224 \mathrm{p}$.

Sáez H., 1997. Resource Degradation, Agricultural Policies, and Conservation in Cuba. Cuban Studies, 27, p. 40-67.

Wotzkow C., 1998. Natumaleza Cubana. Miami, Ediciones Universal, 300 p.

\section{NOTES}

1. Les dates exactes de la "période spéciale» varient selon les auteurs et les approches. Cette période commence au début des années 1990 (1991-1992) avec la période la plus critique entre 1993 et 1997.

2. Il est plus difficile pour les détenteurs de diplômes universitaires d'obtenir de la part du gouvernement cubain les documents leur permettant de sortir du pays, même temporairement, ce qui décourage de plus en plus les jeunes à s'inscrire à l'université.

3. Ces initiatives entrepreneuriales s'inscrivent dans la foulée de la Loi cubaine sur les investissements étrangers (Loi 77). Cette loi, mise en place en 1995, a permis l'établissement de partenariats avec des entreprises étrangères ainsi que les investissements étrangers dans tous les secteurs « à l'exception des services de santé et d'éducation pour la population ainsi que les forces armées » (Alarcon de Quesada, 1995 : 2; voir aussi Morris, 2008). On observe toutefois dans l'exemple présenté ici une interprétation particulière de cette loi, menant à une entorse de ses principes.

4. Parmi les initiatives entreprises à cet égard, Castro a reconnu les objectifs du millénaire en 1993; il a créé un Ministère de l'environnement, des sciences et des technologies (CITMA) en 1994; il a développé une Stratégie environnementale nationale en 1997; et intégré le développement durable économique et social dans la nouvelle constitution. De plus, le pays est reconnu pour ses avancées en matière d'agriculture biologique (Sáez, 1997), la mise en place de six réserves de la biosphère de l'UNESCO et de plus de 236 aires protégées couvrant $22 \%$ du territoire.

5. Bien que le site de la recherche se trouve à une courte distance, $25 \mathrm{~km}$ de l'université, les problèmes de ressources liées au transport font en sorte qu'il est très compliqué pour les chercheurs de s'y rendre et d'y faire de la recherche.

6. Un des moyens pour y parvenir fut entre autres d'établir des cibles de production que les producteurs étaient invités à dépasser moyennant des récompenses (voir Doyon 2007 pour l'exemple de la pêche).

7. Il existe certaines exceptions de pratiques environnementales durables, entre autres avec l'agriculture biologique et urbaine (Premat, 2004; Saenz, 1997). Toutefois, elles ne sont pas non plus exemptes de critiques. 


\section{RÉSUMÉS}

L'éducation postsecondaire et la recherche scientifique sont une des clefs de voûte de la révolution cubaine. Ce secteur offre une notoriété internationale au pays et il est essentiel au processus de construction de l'identité révolutionnaire. Toutefois, il est désormais soumis à d'importantes pressions économiques, menaçant l'avenir de la révolution. Grâce à un exemple ethnographique de la période "post-Castro ", cet article analyse les implications individuelles et institutionnelles de l'insertion de capitaux étrangers mis à la disposition d'un groupe de chercheurs par un projet de développement durable financé par une organisation d'aide internationale au développement.

Education is one of Cuba's revolutionary milestones. It contributes to the country's international notoriety as well as it is central to the revolutionary identity. This sector is now under important economic pressures threatening the Revolution's future. Based on ethnographic data, this paper analyses the individual and institutional implications of foreign capital investments in a local research project funded by an international aid organization in the contemporary "post-Castro" era.

\section{INDEX}

Mots-clés : anthropologie, Cuba, éducation, environnement, révolution

Keywords : anthropology, Cuba, education, environment, revolution

\section{AUTEUR}

\section{SABRINA DOYON}

Sabrina Doyon est Professeur agrégée au département d'anthropologie, Université Laval, Québec, Canada. Ses dernières publications :

Doyon S. et P. Sean Brotherton (eds.), 2008. Mondes socialistes et (post)socialistes. Anthropologie et sociétés, 32, 2-1. 2008.

Doyon S., 2007. Fishing for the Revolution. Transformations and Adaptations in Cuban Fisheries. Mast, 6, 1 :83-108. sabrina.doyon@ant.ulaval.ca 